\title{
Las Representaciones Sociales de calidad educativa presente en los discursos en medios escritos: Un análisis en el contexto del conflicto estudiantil en Chile 2011
}

The Social Representations of Educational Quality in the Discourse of Media Writing: An analysis in the context of students' conflict in Chile, 2011

As Representações Socias de qualidade educativa presentes nos discursos da imprensa escrita: Uma análise no contexto do conflito estudantil no Chile, em 2011

\author{
Ricardo Cerda R. ${ }^{\mathrm{a}}$ y Catalina Opazo B. ${ }^{\mathrm{b}}$
}

aDoctorado en Educación, Pontificia Universidad Católica de Chile. Escuela de Nutrición y Dietética, Facultad de Medicina, Universidad de Chile. Correo electrónico: rjcerda@uc.cl.

${ }^{b}$ Doctorado en Educación, Pontificia Universidad Católica de Chile. CIDE - Facultad de Educación, Universidad Alberto Hurtado. Correo electrónico: copazob@uc.cl. Telf. 56-02-354 5311.

\begin{abstract}
RESUMEN
Este trabajo tiene por objetivo comprender las Representaciones Sociales (RS) de calidad educativa en relación con sus factores de mejoramiento, que aparecen en los discursos de los actores en medios escritos en el contexto del conflicto estudiantil en Chile 2011, a partir del análisis teórico-metodológico de la teoría fundamentada. Se utiliza software Atlas ti v.6.4 para realizar codificación abierta sobre un cuerpo de 29 noticias de medios escritos chilenos, en donde emergen 79 códigos, los cuales sirven de fundamento para la codificación axial. Este proceso iterativo constituyó 16 categorías, del cual surge un modelo explicativo preliminar sobre el fenómeno -entendido como las ideas de calidad, asociadas a nociones y fines de la educación y a estrategias de mejoramiento. Este modelo tiene como base las condiciones causales que definen la calidad de la educación como deficitaria y se relaciona con 3 categorías nucleares: Garantías de calidad, Financiamiento y Evaluación.
\end{abstract}

Palabras clave: calidad educativa, representaciones sociales, medios escritos, conflicto estudiantil.

\begin{abstract}
The aim of this paper is to understand Social Representations of educational quality, in relation to improvement factors, in actors' discourses which appear in written Mass Media within the context of the students' conflict in Chile 2011 from theoretic-methodological analysis of Grounded Theory. Atlas ti v.6.4 was used to make open codification of 29 pieces of news from Chilean written Media, from where 79 codes were created. Through Axial codification, which is an iterative process, 16 categories were built upon the basis of these codes, constituting a preliminary explanatory model about the phenomenon emerged. This phenomenon is defined as the ideas of quality linked to the notions and the aims of education as to improvement strategies. This model is based upon the causal conditions that describe the quality of education as insufficient, and it is related to three key categories: quality guarantees, financing, and assessment.
\end{abstract}

Key words: quality education, social representations, written press, student conflict.

\section{RESUMO}

Objetiva-se, a partir de análise teorico-metodológica da teoria fundamentada, compreender as Representações Sociais (RS) de qualidade educativa em relação a seus fatores de melhoramento, que aparecem nos discursos dos atores da imprensa escrita no contexto do conflito estudantil no Chile, em 2011 no Chile. Utilizou-se software Atlas ti v.6.4 para realizar codificação aberta, de um corpo de 29 notícias da imprensa escrita chilena, de onde emergiram 79 códigos, os quais servem de fundamento para a codificação axial, processo interativo que se constituiu em 16 categorias, de onde surgiu um modelo explicativo preliminar sobre o fenômeno - entendido como as ideias de qualidade associadas às noções e fins da educação e às estratégias de melhoramento. Este modelo baseia nas condições causais que definem a qualidade da educação como deficitária e relaciona-se com três categorias nucleares: Garantia de qualidade, Financiamento e Avaliação.

Palavras chave: qualidade educativa, representações sociais, imprensa escrita, conflito estudantil. 


\section{INTRODUCCIÓN}

Los dos últimos movimientos estudiantiles desarrollados en Chile, han puesto a la Educación en el centro del debate público, la agenda del poder legislativo y de los creadores de políticas. Las movilizaciones del año 2011, lograron una alta adhesión social que se ha expresado en diversas marchas y actos sociales con alta convocatoria. Las demandas del movimiento se han posicionado en el centro de la discusión privada y pública de nuestra sociedad, en torno a los temas de gratuidad, calidad y equidad de la educación y han cuestionado las bases del actual sistema educativo.

Dicho conflicto ha generado discusiones y tomas de posición de distintos actores que han vehiculizado sus discursos en los medios audiovisuales, escritos y electrónicos disponibles en la actualidad, los cuales tiene influencia en la construcción de conocimiento socialmente elaborado y compartido (Umaña, 2002).

El análisis de dichas posiciones y discusiones tiene sentido para comprender cuáles son las Representaciones Sociales (RS) que se encuentran en la base de las distintas acciones de los actores sociales en torno a demandas de transformación o de estabilización del sistema educativo.

Si consideramos que, en la actualidad, los medios de comunicación son uno de los medios más efectivos de socialización política y juegan un rol significativo en el control del flujo de información en la sociedad; y que este flujo se encuentra segmentado en audiencias que poseen ciertos gustos y prácticas de uso de esta información, se hace vital considerarlos para la integración de discursos interpersonales que permiten la interacción social y, por lo tanto, un sustrato necesario para el desarrollo o reproducción del capital social (Khan, 2011). Por otro lado, también se debe tener presente que su impacto resulta significativo a nivel actitudinal, cognoscitivo e ideológico, determinando algunos elementos de los marcos interpretativos que la población utiliza para la comprensión de fenómenos sociales, políticos, económicos, culturales y siendo también una forma de relación con las estructuras de poder, en el sentido de que al ser expresados de un modo en que reducen la complejidad, al entregar un producto terminado, proporciona la ilusión de participar y legitimar el mandato (Suárez, 1999).

Esta idea resulta fundamental para comprender la dimensión pública de los discursos aparecidos en los medios escritos en torno a un determinado conflicto y sus relaciones con determinadas ideologías que se posicionan, ya sea en sectores del campo del poder o de la estructura social (Bourdieu, 1988).

En relación con este sentido y en el contexto en que el conflicto ha estado marcado por una serie de demandas de distintos sectores y de propuestas de solución, se establece tensiones entre posiciones, las cuales son apoyadas por Saberes Expertos que se relacionan de alguna manera con "categorías o representaciones sociales, que sirven para clasificar circunstancias, fenómenos y a los individuos con quienes tenemos que ver; teorías que permiten establecer hechos sobre ellos" (Moscovici, 1986: 472). En la teoría de Moscovici, este proceso estaría determinado por mecanismos de anclaje y objetivación (Umaña, 2002).

De esta manera, cobra especial importancia saber cuáles son las categorías que ayudan a comprender cómo se asocian ciertas propiedades o dimensiones en torno a la educación, calidad y factores de mejoramiento dentro del contexto del conflicto estudiantil. 


\section{MARCO TEÓRICO Y CONCEPTUAL}

\subsection{SABER COMÚN EN SOCIEDAD}

\subsubsection{Representaciones Sociales en Contexto de Conflicto y Reformas}

Para Moscovici (1986) las Representaciones Sociales son imágenes que condensan un conjunto de significados que nos permiten interpretar lo que nos sucede, dar sentido y clasificar circunstancias, fenómenos e individuos con quienes nos relacionamos. Es el conocimiento del sentido común, el cual funciona bajo el supuesto de que es compartido y construido a partir de nuestras experiencias. Incluye conocimientos, creencias y actitudes hacia las cosas, objetos y sujetos. Esta conexión entre los modelos cognitivo, afectivo y procedimentales permite hacer de las RS un puente con el lenguaje, lo ideológico, lo simbólico y lo imaginario de lo social, por tanto, un puente con las conductas y prácticas sociales.

Dado que los movimientos buscan una transformación del sistema educativo y es a partir de esta posibilidad de cambio desde donde se levantan discursos y posiciones que expresan las RS de los diversos grupos sociales, resulta de especial importancia analizarlas, ya que permiten dar sentido a las prácticas que se desarrollan, así como a los hechos y relaciones causales que se establecen (Martinic, 2006). Al respecto, Braslavsky y Cosse (1996) señalan que en las transformaciones educativas -al igual que en otras transformaciones sociales- "intervienen los discursos y las prácticas de numerosos actores" (Braslavsky y Cosse, 19096: 6), determinados por intereses, representaciones y prácticas en diferentes escenarios, aspectos que cristalizados en roles, han mostrado cambios en los últimos años.

Los mismos autores plantean que en contextos de reformas y conflicto surgen 8 tensiones que deberían asumirse como dilemas: 1) Función de la educación, 2) Consenso social y eficiencia, 3) Ejecutar, dinamizar e instrumentar, 4) Conexión con diferentes contextos de referencia, 5) Tiempos políticos, profesionales y burocráticos, 6) Cumplimiento de normas y acción, 7) Necesidad, disponibilidad y utilización de competencias y conocimientos y 8) Tensión en los equipos de trabajo acerca de la búsqueda de profesionalismo en un contexto de ajuste fiscal. Frente a estas, parece existir tres caminos posibles: La innovación, la permanencia del anticambio y los ajustes de mercado; caminos frente a los cuales, se configuran una constelación de actores relativamente estables y cierto grado de especificidad en cada país, con sus propios discursos y posiciones.

El análisis de la posición en el espacio histórico y social de estos actores, con sus discursos vehiculizados en medios de comunicación masiva, tiene sentido porque sus orientaciones y RS en torno a la educación, la calidad y sus factores de mejoramiento, implican el análisis de tres aspectos fundamentales que se encuentran en el corazón del fenómeno que deseamos investigar: La relación entre procesos psicológicos y prácticas sociales, la reificación y legitimación de diferentes sistemas de conocimiento y el rol agencial y de resistencia en la co-construcción de la autoidentidad (Howarth, 2006). Todo ello considerando que las RS no son núcleos sin posibilidades de transformación en la sociedad y que, además, éstas corporizan y definen la experiencia de la realidad, determinando sus límites, su significado y sus relaciones, expresando sentidos particulares que se configuran también en relación con el poder en el orden social, transportando en 
su estado de codificación permanente la polifonía e hibridación de los significados, más que en el sentido de mostrar el significado estable codificado (Howart, 2006).

\subsubsection{Teoría fundamentada}

La Grounded Theory es una de las metodologías más usadas y descritas en ciencias sociales, la cual funda su relevancia en la utilidad empírica, crítica e inductiva que es posible establecer mediante sus procedimientos, los que conectan teoría y práctica de los agentes sociales con la estructura social (Oliver, 2011). En sus orígenes, Glasser y Strauss (1967) plantean que mediante esta metodología-teoría es posible reconstruir un fenómeno a través de tres grandes pasos: codificación abierta, axial y selectiva, los que permiten interpretar la acción social, identificar el valor que se le da al conocimiento común de la experiencia y, ya que los seres sociales orientan sus prácticas hacia otros, actuar en función del valor que tienen para ellos.

La teoría permite explicar la conducta a partir del interaccionismo simbólico, donde la acción involucra varia formas de comunicación, verbal y no verbal, en la cual la noción simbólica es intrínseca a esta perspectiva (Goulding, 2003). Esto genera un enfoque en el cual los individuos sociales nacen en una sociedad donde existen símbolos y objetos ya construidos y donde estos, al interactuar con ellos, los reconstruyen.

\subsection{SABER EXPERTO VEHICULIZADO EN PUBLICACIÓN E INFORMES}

\subsubsection{Nociones generales de Calidad: Raíces de la definición general de Calidad}

El concepto de Calidad ha sido usado históricamente en múltiples ámbitos y, en la actualidad, ocupa un lugar privilegiado en el ámbito del desarrollo de organizaciones y de la economía, los negocios y el marketing (Reeves \& Bednar, 1994). La Calidad se ha planteado como un concepto multifacético y que frecuentemente lleva a confusión en distintos ámbitos. Se ha planteado que el significado de la Calidad no puede ser definido cuando se busca establecer una relación entre distintas variables, con un constructo que mirado como una variable dependiente continuamente se modifica (Cameron \& Whetten, 1993).

Reeves y Bednar (1994) proponen que las primeras discusiones respecto al concepto provienen de los filósofos griegos, como un concepto relacionado con un ideal, llamado "Areté", es decir, Excelencia. El concepto, según esta vertiente, puede variar según el contexto y el fenómeno analizado; así, en una carrera de caballos, por ejemplo, el concepto tiene relación con la velocidad, con la fuerza, con el carro; en cambio, una persona es excelente en términos de las connotaciones morales, intelectuales, físicas, prácticas que implica para un determinado asunto. Para Platón, el "Areté" es definido en términos absolutos: el bien en su más alta forma, la mayor idea de todas.

Actualmente, se define la Calidad en términos de excelencia, como la manera de investir con la mejor habilidad y esfuerzos posibles para producir el resultado más fino y admirable posible. Se plantea que este tipo de definiciones poseen el problema que la experiencia directa muchas veces no se relaciona con las abstracciones intelectuales.

Rondando el siglo XVIII, en el ámbito de los negocios, otra corriente define Calidad como un valor; es decir, dado que la Calidad no es en sentido popular lo mejor en términos absolutos, su definición se circunscribió a términos relativos dentro de un mercado y 
bajo la experiencia de un consumidor, en la que se incluye el precio como una variable importante para evaluar los productos.

En el primer tercio del siglo XX, la definición que entró en juego fue la Calidad conforme a especificaciones o, en otras palabras, la creación de estándares por consenso en determinados productos, concepto desarrollado en Estados Unidos producto de la necesidad de vender piezas intercambiables en la industria de armamentos. Siguiendo la formula: si la parte no reúne las especificaciones no puede ser intercambiable y si no es intercambiable no es una buena pieza. Dicha formula se intensificó con el desarrollo de la industria automotriz, en donde el producto se masificó. Con la implementación del control de ingeniería de procesos, se logró asegurar en parte que las piezas pudieran lograr las especificaciones y entonces se introduce a la idea de cumplimiento de ciertas características: el control del proceso al menor costo posible. De esta manera, la Calidad en los productos industriales se transformó de algo que no podía ser cuantificable en algo que sí lo podía ser y, además, en algo que podía ser controlado y comparable entre mercados.

En la actualidad, la definición dominante de Calidad corresponde a si un producto o servicio es apropiado o no o si excede las expectativas del cliente, definiciones que han sido utilizadas en otros campos, como el educativo.

\subsubsection{Construcción de las Nociones de Calidad Educativa}

La discusión sobre calidad de educación puede circunscribirse a diferentes marcos y niveles. Un primer nivel corresponde a la función social atribuida a la educación, la que, a su vez, se vincula a diversas visiones de desarrollo social y humano y con definiciones de los fines éticos y políticos de la educación. Desde la función clásica de transmisión de los valores que dan cohesión a la sociedad y mantención de las estructuras sociales, la calidad educativa estará dada por su capacidad para mantener los equilibrios macrosociales y su efectividad en la transmisión de normas, valores, actitudes y conocimientos que favorezcan la adaptabilidad de los sujetos a su entorno y a la estructura social.

Por otro lado, desde la teorías críticas de la Reproducción Social, la función de la educación es favorecer la concientización y la integración social por medio de procesos de develamiento de las desigualdades y de empoderamiento de las nuevas generaciones (Freire, 1986). Ello requiere desarrollar el pensamiento crítico y la creatividad en los sujetos, pero también transformar las estructuras sociales que perpetúan la desigualdad. Desde este enfoque, la Calidad de la educación se asocia a su capacidad para empoderar a los individuos y favorecer la transformación social.

En la literatura sobre Calidad en Educación es posible encontrar diversas perspectivas para definir este concepto, entra las que es posible destacar algunas relevantes. En primer lugar, desde el enfoque de derechos se enfatiza el cumplimiento del derecho a la educación y el pleno desarrollo de la persona humana, cuya garantía es posible en el ejercicio de otros derechos fundamentales. Desde esta óptica, la educación de calidad tiene como cualidad fundamental el respeto de los derechos de las personas, especialmente el derecho a la no discriminación y a la plena participación, el que debe expresarse en garantías de gratuidad y obligatoriedad. La UNESCO (2007) identifica cinco criterios de una educación de calidad: la equidad, debiendo "ofrecer los recursos y ayudas necesarias para que todos los estudiantes alcancen los máximos niveles de desarrollo y aprendizaje" (UNESCO, 2007: 34), la pertinencia y la relevancia, de acuerdo a las cuales los contenidos 
y estrategias de la educación debe ser significativos, facilitando la apropiación de los contenidos de la cultura y, a la vez entregar, las competencias necesarias para participar e integrarse, según las finalidades que la sociedad le otorga a la educación; finalmente, la eficiencia y eficacia, asociada al uso adecuado de los recursos y el logro de determinados estándares de aprendizaje. Este enfoque se expresa también en la perspectiva de la educación inclusiva, cuya principal aspiración es asegurar el derecho a una educación de calidad para toda la población. Desde este enfoque se entiende que los grandes fines de la educación son los mismos para todos los niños y niñas, sean cuales fueren los problemas que enfrentan en su proceso de desarrollo y de aprendizaje. Hay cuatro elementos que han contribuido a dar forma al concepto de inclusión (UNESCO, 2005): visión del desarrollo como proceso, la identificación y la eliminación de barreras al aprendizaje, la presencia, participación y logros (resultados) de todos los estudiantes y el énfasis en los grupos de estudiantes en riesgo de marginación, exclusión o bajos resultados.

Una perspectiva complementaria, también difundida por UNESCO, enfatiza en una concepción holística. Esta perspectiva fue construida basada en cuatro conferencias realizadas entre el año 2008 y 2009, cuyos resultados indican cómo esta concepción podría ayudar a solucionar los problemas del mundo contemporáneo en los que la educación podría influir, siempre que se formulen políticas integradas basadas en una concepción holística e equitativa del sistema educativo (UNESCO, 2008). En el informe se planteó que la educación tiene como finalidad el desarrollo humano a través del ciclo vital con miras a disminuir las inequidades, favorecer la inclusión, la cohesión social y la justicia social, lo cual implica mirar la educación en un sentido más amplio que sólo el desarrollo de habilidades cognitivas, sino también el desarrollo de valores y habilidades a través de la educación formal y no formal, a lo largo de la vida y donde los profesores poseen un rol irreemplazable. En el mismo sentido de integralidad, pero con un foco distinto, la UNICEF (2000) plantea que la calidad en educación corresponde también a otras dimensiones, tales como lograr educar niños saludables y bien nutridos que se encuentren dispuestos a aprender en ambientes seguros y saludables, sensibles a condiciones de género y que tengan los recursos y facilidades para desarrollarlas, que cuente con currículum que les permitan su desarrollo, con profesores bien entrenados y con foco en los niños, que se cuente con clases bien manejadas, con el foco en el desarrollo de conocimientos, habilidades y actitudes que sean coherentes con los objetivos nacionales y con una adecuada participación en sociedad.

Una tercera perspectiva corresponde a la propuesta por la teoría del Capital Humano, según la cual la educación debe desarrollar las competencias requeridas para que los sujetos logren insertarse productivamente al mundo del trabajo, lo que implica un importante vínculo entre la educación y el mercado, con foco en la formación en "destrezas productivas pertinentes" (Ottone y Hopenhayn, 2007), favoreciendo el desarrollo productivo general y, a la vez, la igualdad de oportunidades mediante mecanismos de movilidad social meritocrática.

\subsubsection{Los procesos de mejoramiento y aseguramiento de la Calidad}

Desde el punto de vista de la política educativa, un aspecto clave en la discusión de la última década ha sido la generación de mecanismos y procesos que permitan asegurar la Calidad Educativa. Ello implica construir un concepto de Calidad que sea operacionalizable, 
observable y medible y permita "fortalecer la capacidad de gestión de los administradores públicos y privados de la educación para transformarlos en contrapartes efectivas de las nuevas políticas y prácticas de rendición de cuentas y control de calidad" (Claro y Espínola, 2007: 52). Este concepto de Calidad ha estado ligado, fundamentalmente, a resultados de aprendizaje evaluables de forma estandarizada.

Estas nociones están a la base de lo que se ha llamado Reforma Basada en Estándares (RBE), consistente en un modelo que se sustenta en la definición precisa de estándares exigibles a los diferentes actores del sistema educativo, quienes reciben sanciones, reconocimientos o premios asociado su logro. De acuerdo a esta perspectiva "se trata de que los participantes del sistema educativo sepan lo que se espera de ellos y tengan metas en sus respectivos ámbitos de acción (...) Básicamente se trata de un circuito de rendición de cuentas compuesto por los estándares, la evaluación y las consecuencias, el cual se complejiza según las necesidades de los países" (Claro y Espínola, 2007: 57).

\section{PROBLEMA, PREGUNTAS E HIPÓTESIS Y OBJETO DE ESTUDIO}

\subsection{PROBLEMA}

Durante el año 2011, la sociedad Chilena ha sido testigo del mayor movimiento estudiantil que recuerde. Las demandas del movimiento han ubicado en el centro de la discusión aspectos tales como la gratuidad, calidad y equidad del sistema educativo y han cuestionado las bases fundacionales del actual modelo educativo. El movimiento ha dado pie a una gran proliferación de discusiones y tomas de posición en los medios públicos por una gran multiplicidad de actores, quienes analizan, cuestionan y proponen las condiciones y posibilidades de mejoramiento del conflicto y del sistema educativo.

En el marco de este debate, la discusión sobre la calidad educativa es fundamental, la cual aparece en los discursos de tipo evaluativo en relación con juicios de expectativas a nivel nacional e internacional y en las expresiones sobre demandas y propuestas de mejoramiento y cambio de la educación chilena. Estos discursos sobre calidad educativa no aparecen de forma aislada, sino que asociados a otras discusiones que han adquirido mayor notoriedad y que ponen como temas centrales del debate sobre educación las discusiones respecto a la demanda por gratuidad -especialmente en la educación superior-, el cuestionamiento al lucro y al sistema de provisión mixta.

La discusión sobre calidad educativa parece ser un aspecto base, en la medida que define un propósito que podría marcar cursos de acción que permitan encontrar vías de salida del actual conflicto, sin embargo, ha quedado oculto entre las discusiones más contingentes. Este estudio busca develar y analizar las diferentes interpretaciones y RS sobre Calidad Educativa; y cómo estas diversas ideas se relacionan con las demandas y propuestas de mejoramiento que se han expresado en el debate público durante el desarrollo del movimiento estudiantil.

Basado en lo anterior, se formularon las siguientes preguntas de investigación: ¿Qué representaciones sociales de calidad aparecen en los discursos de los actores en medios escritos en el contexto del conflicto estudiantil chileno 2011? ¿Qué factores de mejoramiento de la calidad son propuestos en los discursos en medios escritos en el contexto 
del conflicto estudiantil chileno 2011? ¿Cómo se relacionan los diversos factores de mejoramiento con las nociones de calidad identificadas?

\subsection{HIPÓTESIS}

Las RS de calidad se construyen en la articulación de las diversas perspectivas sobre fines de la educación, definiciones de calidad y factores de mejoramiento. Es posible establecer relaciones entre fines de la educación, definiciones de calidad y factores de mejoramiento. Dependiendo de las relaciones diferenciadas que se establecen entre fines de la educación, definiciones de calidad y factores de mejoramiento, es posible identificar cursos de acción en diferentes niveles, los que van de lo micro a lo macro.

\subsection{OBJETO}

Las RS de calidad en relación a sus factores de mejoramiento de la educación que aparecen en los discursos de los actores en medios escritos en el contexto del conflicto estudiantil en Chile 2011.

\subsection{OBJETIVO DE LA INVESTIGACIÓN}

El objetivo del presente trabajo es comprender la RS de calidad educativa, en relación con sus factores de mejoramiento, que aparecen en los discursos de los actores en medios escritos en el contexto del conflicto estudiantil en Chile 2011.

\section{DISEÑO, METODOLOGÍA Y PROCEDIMIENTOS}

El diseño corresponde a un estudio de tipo cualitativo de análisis comparativo constante de discursos expresados en medios escritos entre Julio y Octubre del 2011, tendiente a reconstruir la teoría fundamentada expresada respecto a las relaciones entre nociones de calidad y factores de calidad en torno al conflicto estudiantil.

\subsection{MUESTREO TEÓRICO}

Dado el objetivo del estudio, se seleccionó un cuerpo de documentos que permitiera analizar discursos en torno a las nociones de calidad en educación y sus factores de mejoramiento, el cual se constituyó a partir de 29 documentos disponibles en las páginas web de medios de prensa, escritos y radiales y otros organismos relacionados con la educación, entre los meses de julio y noviembre del 2011. Estos documentos fueron analizados durante el proceso de codificación abierta, para identificar si los textos abordaban las temáticas buscadas. Se elaboró una tabla que relacionó los temas abordados, clasificados en 3 categorías: nociones y principios de la educación y calidad; factores a nivel micro y factores a nivel macro; con 4 tipos de actores: gobierno; oposición; sociedad civil a favor del movimiento; y columnistas, editores, expertos y académicos. Se determinó si los textos cubrieron o no las temáticas buscadas y si el muestreo teórico fue equilibrado en la representación de distintos actores y medios en torno a las hipótesis planteadas previamente. 
En la Tabla 1 se muestra la cobertura de los distintos documentos de las temáticas y de los actores considerados en el análisis posterior. Se aprecia que los 29 documentos seleccionados lograron cubrir tanto las temáticas buscadas como las posiciones de algunos actores, utilizando la dirección de sentido del movimiento estudiantil como principio de clasificación (Pro movimiento/ Pro Gobierno). Donde este principio no fue tan marcado se rotuló como "Neutral". Se aprecia que el mayor número de documentos abordan las temáticas a nivel macro y nociones y principios en torno a la calidad y educación que se persigue. Por otro lado, se observa que son las columnas de opinión categorizadas como neutrales, las que abordan en mayor medida las tres temáticas mencionadas. Por último, cabe agregar que la principal fuente de discurso de los actores de gobierno y sociedad civil, provino de declaraciones públicas.

Tabla 1. Cobertura de las temáticas y de los actores considerados con el muestreo teórico de documentos

\begin{tabular}{|c|c|c|c|c|c|c|c|}
\hline \multirow[t]{2}{*}{ Tema/Actor } & \multirow[t]{2}{*}{ Gobierno } & \multirow[t]{2}{*}{ Oposición } & \multirow{2}{*}{$\begin{array}{c}\text { Sociedad } \\
\text { civil pro } \\
\text { movimiento* }\end{array}$} & \multicolumn{3}{|c|}{$\begin{array}{c}\text { Noticias, columnistas, editores, } \\
\text { expertos y académicos }\end{array}$} & \multirow[t]{2}{*}{$N^{o}$} \\
\hline & & & & $\begin{array}{l}\text { Pro } \\
\text { movimiento }\end{array}$ & $\begin{array}{c}\text { Pro } \\
\text { Gobierno }\end{array}$ & Neutral & \\
\hline $\begin{array}{c}\text { Nociones y } \\
\text { principios } \\
\text { de calidady } \\
\text { educación }\end{array}$ & $\begin{array}{c}q \\
(1)^{* * * *}\end{array}$ & $\begin{array}{l}b, f \\
(2)\end{array}$ & $c, d, e, k, u$ & $\begin{array}{l}i, n, r \\
(3)\end{array}$ & $\begin{array}{c}G \\
(1)\end{array}$ & $\begin{array}{l}a, j, r \\
s, x \\
(5)\end{array}$ & 17 \\
\hline $\begin{array}{c}\text { Factores } \\
\text { Nivel Micro: } \\
\text { Aula, Escuela- } \\
\text { Universidad- } \\
\text { Instituto }\end{array}$ & $\begin{array}{l}l, w \\
(2)\end{array}$ & $\begin{array}{c}f \\
(1)\end{array}$ & $\begin{array}{l}e \\
(1)\end{array}$ & $\begin{array}{c}r \\
(1)\end{array}$ & $\begin{array}{l}g, m \\
(2)\end{array}$ & $\begin{array}{c}a^{* *}, j, p, \\
v, a b, a c \\
\text { (6) }\end{array}$ & 13 \\
\hline $\begin{array}{c}\text { Factores } \\
\text { Nivel Macro: } \\
\text { Estructura, } \\
\text { Sistema, Políticas, } \\
\text { evaluación, } \\
\text { estándares }\end{array}$ & $\begin{array}{l}l, q \\
(2)\end{array}$ & $\begin{array}{l}b, f \\
(2)\end{array}$ & $c, d, e, k, u$ & $\begin{array}{l}i, n, r, u, y \\
\quad(5)\end{array}$ & $\begin{array}{c}g, h, m \\
\quad(3)\end{array}$ & $\begin{array}{c}a, j, o, p, \\
r, s, t, x, \\
z, a a \\
\quad(10)\end{array}$ & 27 \\
\hline Total & 5 & 5 & 11 & 9 & 6 & 21 & 29 \\
\hline
\end{tabular}

* Incluye confederación de estudiantes de universidades y secundarios. **Las letras fueron asignadas a cada documento en orden correlativo, para identificar su contribución a la grilla. *** Los números entre paréntesis indican el número de documentos en el cruce de filas temáticas con columnas de actores.

Estos documentos, a su vez, provenían de los siguientes medios online: El Mercurio (8), El Mostrador (4), declaraciones públicas en medios diversos (4), La Tercera (2), Universidad de Chile (2), BBC (1), CIPPER (1), Cooperativa (1), Dínamo (1), Educación 2020 (1), El Post (1), Idea País (1), Revistas bUSinnes Chile (1). Estos medios hicieron disponibles los discursos de los siguientes actores sociales: Periodistas (7), Académicos (6), Columnistas no académicos (3), Estudiantes (3), Concertación (2), Ciudadanos (2), Actor no reconocido (2), Experto extranjero (2), Experto Asesor de gobierno (1), Editor de Diario (1). 


\subsection{PROCESO DE VALIDACIÓN EN LA CODIFICACIÓN}

El proceso de codificación se desarrollo en el programa Atlas ti versión 6.4, mediante la creación de unidades hermenéuticas y la aplicación de las fases recomendadas por la teoría fundamentada por dos investigadores, que se dividieron el cuerpo de documentos para su análisis y luego lo fundieron en una gran unidad. Antes de realizar la codificación abierta, los investigadores analizaron cinco textos del corpus descrito en él y, posteriormente, se analizó su nivel de concordancia en la identificación de unidades de sentido y la utilización de códigos en relación a si estos fueron usados de manera pertinente, efectiva, relevante y según criterio de modificación del sentido final de una unidad de sentido. Los resultados del análisis indicaron niveles moderados a altos de concordancia en la identificación de unidades de sentido y niveles moderados, en la interpretación de los textos e incidentes que derivaron en la selección o elaboración de códigos, por lo que se procedió a elaborar un protocolo con pasos y criterios que los investigadores siguieron en las siguientes fases del estudio. El protocolo consistió en los siguientes pasos basado en literatura existente (Flores, 2009; Vasilachis de Gialdino, 2006; Strauss y Corbin, 2002): a) Selección de fragmentos de los textos para identificar unidades de sentido en relación con las preguntas de investigación, los objetivos y algunas categorías provisorias elaboradas previamente, b) Decisión de utilización de categorías provisorias, códigos en vivo o código abierto según correspondiera, c) Definición o redefinición de códigos y d) Elaboración de memos categoriales, metodológicos de limitaciones y problemas teóricos y metodológicos de codificación abierta y memos de relaciones emergentes entre códigos y posibles categorías.

El protocolo utilizado para aplicar la teoría fundamentada por los dos investigadores no estuvo exento de dificultades, debido a la necesidad constante de explicitación y discusión de criterios de interpretación de las unidades de sentido por grillas epistemológicas de la historia previa de los investigadores frente al fenómeno. Producto de la aplicación del protocolo, se pudo llegar a un nivel aceptable de concordancia.

\subsection{CODIFICACIÓN ABIERTA}

Para efectos de esta investigación, se utilizaron códigos en vivo, códigos abiertos y códigos preelaborados por los investigadores en una fase anterior, los que, sumados a la fase de validación, dio como resultado una base de sesenta códigos. Esta base fue revisada por ambos investigadores, verificando sus definiciones y su coherencia con los objetivos de la investigación, ajustándolos y verificando su funcionalidad a la hora de interpretar el sentido de los textos descritos. En el proceso de codificación abierta y posterior a la fusión de algunos códigos se obtuvieron setenta y nueve códigos. Se crearon además cincuenta y seis Memos y se identificaron doscientas setenta y seis unidades de sentido o Quotations.

\subsection{CODIFICACIÓN AXIAL}

En este proceso se buscó reorganizar las relaciones entre categorías y códigos en función de las propiedades y dimensiones emergentes del proceso anterior, por medio de la estrategia de identificación de relaciones condicionadas, lo que además permitió 
reconocer categorías o ejes centrales que permiten ordenar múltiples sentidos del modelo o teoría en construcción. En este proceso, se siguieron los siguientes pasos:

- Lectura iterativa de definiciones de códigos y memos, haciendo notas que permitieron la agrupación de familias.

- Agrupación de códigos pertenecientes a sentidos similares, en los casos pertinentes. Otros mantuvieron su sentido inicial.

- Elaboración de 16 categorías o grandes familias de agrupación, las cuales se mencionan y definen en la Tabla 2, que sirvieron de base para el análisis de relaciones condicionales y de propiedades y dimensiones.

- Construcción de un modelo de relaciones condicionadas, reconociendo que las categorías correspondieron a condiciones (contextuales, causales e intervinientes), acciones o interacciones y consecuencias. Para esto, los investigadores relacionaron subcategorías por medio de oraciones con categorías y luego se buscaron claves en las unidades de sentido y en los memos que permitieran relacionar las grandes categorías.

- Identificación de categorías centrales a partir del análisis de frecuencia de unidades de sentido y de los esquemas de relaciones condicionadas.

Tabla 2. Definición de las categorías preliminares de clasificación de los códigos

\begin{tabular}{|c|c|c|c|}
\hline $\mathrm{N}^{\mathrm{o}}$ & CATEGORÍA & CÓDIGOS ASOCIADOS (No)/QUOTATIONS (No): [Códigos] \\
\hline 1 & $\begin{array}{c}\text { Acciones Macro } \\
\text { Evaluación }\end{array}$ & $\begin{array}{c}\text { Codes (5)/Quotation(s) 46: [C.Evaluación como factor de calidad] } \\
\text { [E.Comparación internacional de calidad] [E.Formas de evaluación } \\
\text { de la calidad de la educación en el sistema educativo] [E.Indicadores } \\
\text { y estándares de calidad] [E.Visión evaluativa de la calidad de la } \\
\text { educación en chile] }\end{array}$ \\
\hline 2 & $\begin{array}{c}\text { Acciones Macro- } \\
\text { Micro Investigación }\end{array}$ & $\begin{array}{c}\text { Codes (1)/Quotation(s)5 : [Investigación como factor de calidad] } \\
\text { Acciones Macro } \\
\text { Garantías }\end{array}$ & $\begin{array}{c}\text { Codes (5)/Quotation(s) 66:[F.Fortalecimiento de la educación } \\
\text { pública] [F.Garantía de calidad] [F.Mecanismos estatales asociados a } \\
\text { calidad educativa] [F.Relación entre políticas públicas y la calidad de } \\
\text { la educación] [F.Rol del Estado en la calidad] }\end{array}$ \\
\hline 5 & $\begin{array}{c}\text { Acciones Macro } \\
\text { Institucionalidad y } \\
\text { Actores }\end{array}$ & $\begin{array}{c}\text { Codes (6)/Quotation(s) 27:[C.Participación ciudadana como factor de } \\
\text { calidad] [C.Trayectoria y tradición como factor de calidad] [D.Estrategia } \\
\text { de mejoramiento asociada a educación técnico profesional] [F.Forma de } \\
\text { organización entre entidades educativas] [F.Institucionalidad y Actores] } \\
\text { [X. Aportes Universidad de Chile al país] }\end{array}$ \\
\hline 6 & $\begin{array}{c}\text { Acciones Macro } \\
\text { Provisión }\end{array}$ & $\begin{array}{c}\text { Codes (6)/Quotation(s)37:[C.Dependencia administrativa de los } \\
\text { establecimientos educativos como factor de calidad] [F.Forma de } \\
\text { organización entre entidades educativas] [F.Fortalecimiento de } \\
\text { la educación pública] [F.Políticas y regulación de la provisión] } \\
\text { [G.Organización del Sistema Educativo] [H. Privado] }\end{array}$ \\
\hline 7 & $\begin{array}{c}\text { Acciones Macro } \\
\text { Regulación }\end{array}$ & $\begin{array}{c}\text { Codes (3)/Quotation(s): 37:[C.Regulación y control como factor } \\
\text { de calidad] [D.Estrategias de regulación y control para mejorar la } \\
\text { calidad] [E.Formas de evaluación de la calidad de la educación en el } \\
\text { sistema educativo] }\end{array}$ \\
\hline
\end{tabular}




\begin{tabular}{|c|c|c|}
\hline 8 & $\begin{array}{l}\text { Acciones Micro } \\
\text { Docente-Aula }\end{array}$ & $\begin{array}{l}\text { Codes (15)/Quotation(s)38:[C. Desempeño docente como factor de } \\
\text { calidad] [C.Capacitación como factor de Calidad] [C.Escritura como } \\
\text { factor de calidad] [C.Evaluación docentes como factor de calidad] } \\
\text { [C.Formación inicial docente como factor de calidad] [C.Gestión del } \\
\text { aprendizafe como factor de calidad] [C.Práctica de aula como factor } \\
\text { de calidad] [C.Rol del profesor en la calidad] [C.Selección de futuros } \\
\text { profesores como factor de calidad] [C.Status profesor como factor de } \\
\text { calidad] [C.Vocación y compromiso como factor de calidad] [E.Calidad } \\
\text { asociada a cambio] [G.Necesidad nacional de formación de profesores] } \\
\text { [H. Entornos, Arquitectura y Materiales como factores de calidad] } \\
\text { [X.Escuela como espacio de contención y formación] }\end{array}$ \\
\hline 9 & $\begin{array}{l}\text { Acciones Micro } \\
\text { Gestión Escolar }\end{array}$ & $\begin{array}{l}\text { Codes (6)/Quotation(s)23:[C.Accountability como factor de calidad] } \\
\text { [C.Autonomía escolar como factor de calidad] [C.Capacitación } \\
\text { como factor de Calidad] [C.Evaluación como factor de calidad] } \\
\text { [C.Gestión de recursos como factor de calidad] [C.Gestión escolar } \\
\text { como factor de calidad] }\end{array}$ \\
\hline 10 & $\begin{array}{l}\text { Acción Micro } \\
\text { Estudiante-Familia }\end{array}$ & $\begin{array}{l}\text { Codes (2)/Quotation(s) 3:[C.Compromiso alumnos como factor de } \\
\text { calidad] [C.Familia como factor de calidad.] }\end{array}$ \\
\hline 11 & $\begin{array}{c}\text { Condiciones } \\
\text { Causales }\end{array}$ & $\begin{array}{l}\text { Codes (14)/Quotation(s)126: [C.Calidad de las instituciones de Ed } \\
\text { Superior como factor de calidad] [C.Evaluación como factor de } \\
\text { calidad] [C.Financiamiento como factor de calidad] [C.Reformas } \\
\text { como factor de Calidad] [E.Comparación internacional de calidad] } \\
\text { [E.Visión evaluativa de la calidad de la educación en chile] [F.Rol } \\
\text { del Estado en la calidad] [F.Sistema de financiamiento del sistema } \\
\text { educativo] [G.Calidad y (des) igualdad] [G.Organización del Sistema } \\
\text { Educativo] [H. Privado] [H.Cobertura] [H.Segregación escolar] [X. } \\
\text { Efectividad de la reformas en la calidad] }\end{array}$ \\
\hline 12 & $\begin{array}{l}\text { Condiciones } \\
\text { Contextuales }\end{array}$ & $\begin{array}{c}\text { Codes (3)/Quotation(s)17:[G.Cambios sociales y factores } \\
\text { contextuales] [G.Demandas de la sociedad a la educación] } \\
\text { [G.Preferencias sociales y culturales de carreras universitarias y } \\
\text { técnicas] }\end{array}$ \\
\hline 13 & $\begin{array}{l}\text { Condiciones } \\
\text { Intervinientes }\end{array}$ & $\begin{array}{c}\text { Codes (3)/Quotation(s)5: [G.Diversidad educativa] [X.Niveles } \\
\text { educativos] [X.Tiempo como factor de implementación de políticas.] }\end{array}$ \\
\hline 14 & Noción de Calidad & $\begin{array}{c}\text { Codes (9)/Quotation(s)33:[A.Calidad versus gratuidad] [B.Calidad } \\
\text { como concepto multivariado] [B.Calidad como educación } \\
\text { integral] [B.Calidad como excelencia] [B.Calidad como éxito } \\
\text { social] [B.Calidad como proceso] [B.Calidad como producto] } \\
\text { [B.Cuestionamiento a la noción de calidad] [E.Indicadores y } \\
\text { estándares de calidad] }\end{array}$ \\
\hline 15 & $\begin{array}{l}\text { Noción de } \\
\text { Educación }\end{array}$ & $\begin{array}{l}\text { Codes (7)/Quotation(s) 35:[A. Educación como comprensión y } \\
\text { transformación del entorno] [A.Educación como búsqueda de la verdad] } \\
\text { [A.Educación y movilidad social] [A.Noción de educación como bien } \\
\text { de consumo individual] [A.Noción de educación como derecho y bien } \\
\text { público] [A.Noción de educación como desarrollo integral] [A.Noción } \\
\text { de Educación como formación de capital humano] }\end{array}$ \\
\hline
\end{tabular}




\begin{tabular}{|c|c|c|}
\hline 16 & Principios & Codes (5)/Quotation(s) 54:[G.Calidad y (des) igualdad] \\
& $\begin{array}{c}\text { Orientadores } \\
\text { Propósitos } \\
\text { Educación }\end{array}$ & [G.Demandas de la sociedad a la educación] [G.Fines de la \\
& educación] [H. Privado] [H.Gratuidad] \\
\hline
\end{tabular}

\section{RESULTADOS}

A partir del proceso de codificación axial es posible establecer las relaciones que definen el fenómeno en estudio, el cual se entiende como las ideas de calidad que expresan los actores sociales, asociadas a las nociones y fines de la educación y a estrategias de mejoramiento. En los esquemas que se presentan a continuación se ilustran las relaciones entre las condiciones (causales, contextuales e intervinientes), las que dan cuenta de las circunstancias y situaciones que permiten contextualizar el fenómeno en estudio; las relaciones entre las acciones o interacciones que dan cuenta de diversos factores de mejoramientos que se expresan como posibles estrategias de transformación y propuestas; y las relaciones entre consecuencias, que indica lo que sucede como resultado de las acciones y se expresa en las aspiraciones sobre educación y calidad.

Figura 1. Condiciones

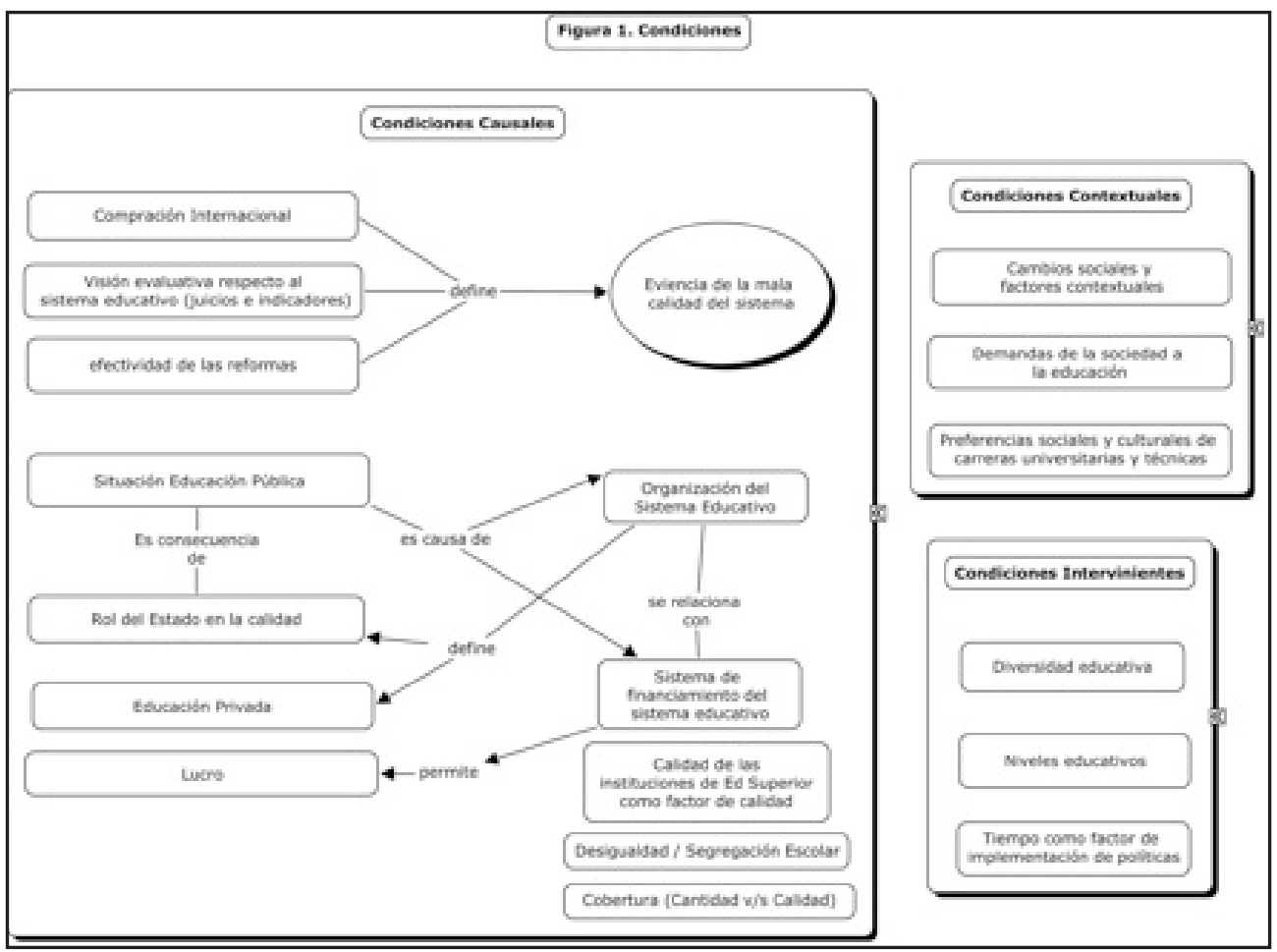


Las condiciones causales (Fig.1) quedan expresadas, primero, en expresiones que evidencian la mala calidad del sistema educativo, a través de las comparaciones internacionales, juicios e indicadores que expresan una visión evaluativa del sistema y el juicio sobre la efectividad de las reformas. En un segundo nivel, se establecen relaciones entre características de la organización y los mecanismos de financiamiento del sistema educativo, los que van articulando la forma en cómo se han configurado algunos aspectos que están en el núcleo del debate, tales como la situación (desmejorada) de la educación pública, el efecto de la incorporación de una lógica de privatización, la posibilidad de lucrar y el consiguiente rol que asume el Estado en este contexto. Por último, aparecen algunos aspectos discretos que también pueden considerarse como condiciones causales, entre los que se distingue la calidad de las instituciones educativas, en particular las de educación superior, la crítica a la situación de desigualdad y segregación que podría (o no) redundar en una mala calidad educativa y una crítica respecto a cobertura, entendida como foco en la cantidad y no en la calidad.

También se identifican algunas condiciones contextuales, entre las que se distinguen las demandas sociales a la educación y los cambios sociales y factores contextuales que corresponden a códigos que identifican tanto las demandas de las transformaciones macro sociales, culturales y económicas, como aquellas vinculadas a los contextos inmediatos de los estudiantes y sus familias. También se considera que preferencias sociales y culturales de carreras universitarias y técnicas son una condición contextual que afecta la decisión de potenciales profesores.

Las condiciones intervinientes identificadas corresponden a situaciones de diversidad educativa, las distinciones que se establecen entre los diversos niveles educativos y el factor temporal en la implementación de políticas.

Figura 2. Acciones e interacciones

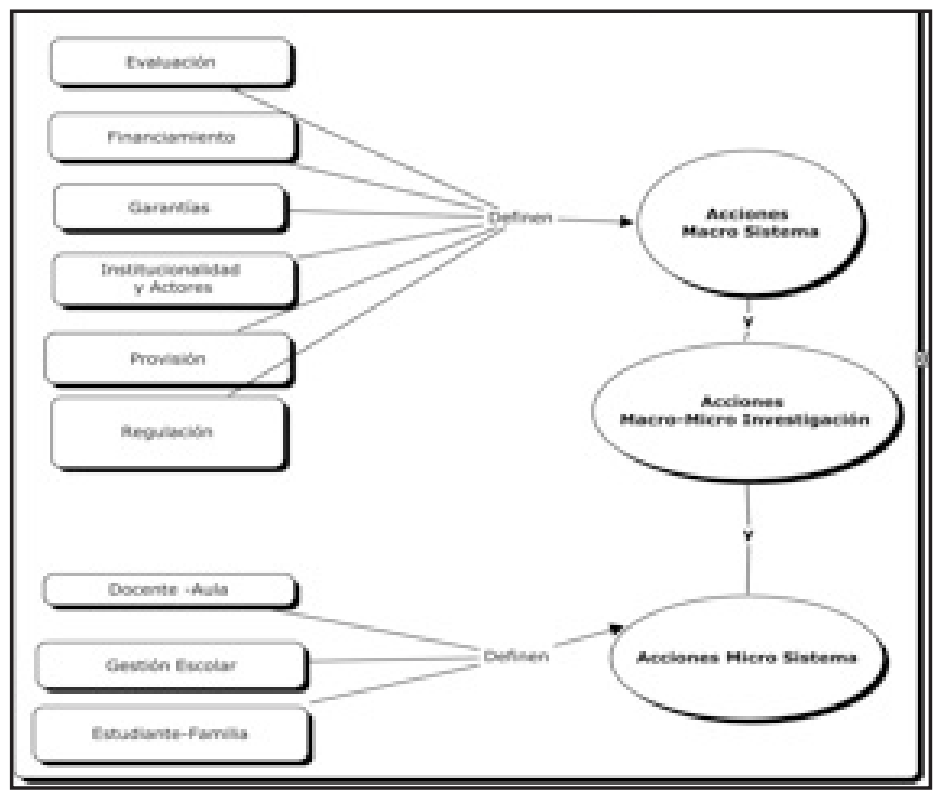


Las acciones e interacciones (Fig. 2) fueron identificadas a partir de los factores de mejoramiento e indican aquellos ámbitos en los que se requiere implementar transformaciones o hacer ajustes que permitirían mejorar la calidad de la educación. Pueden clasificarse en acciones macro y micro, más una categoría -investigación- que vincula ambos niveles. A nivel macro se identificaron los siguientes elementos: Evaluación, Financiamiento, Garantías (de una educación de calidad), Institucionalidad y actores, Sistema de provisión y mecanismos de regulación y control. A nivel micro, se identificaron acciones en asociadas a docentes y prácticas de aula, a aspectos de la gestión y el liderazgo escolar y al involucramiento del estudiante y la familia.

\section{Figura 3. Consecuencias}

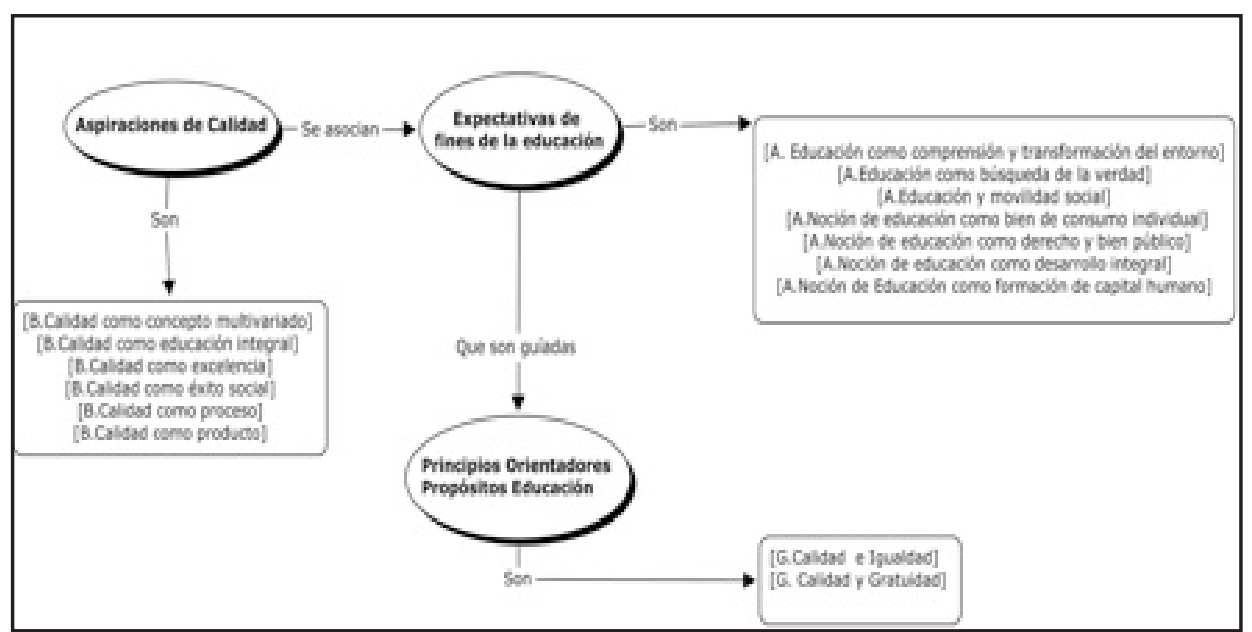

Por último, como consecuencia se identificaron 3 núcleos centrales (Fig.3). El primero corresponde a aspiraciones de calidad que se expresan en las diversas nociones acerca de este concepto que aparecen en los discursos de los actores, las que a su vez se asocian a expectativas de fines de la educación, expresados en nociones y propósitos atribuidos a la educación. Éstos son guiados por principios orientadores más generales tales como la igualdad y la gratuidad. 
Figura 4. Categorías centrales

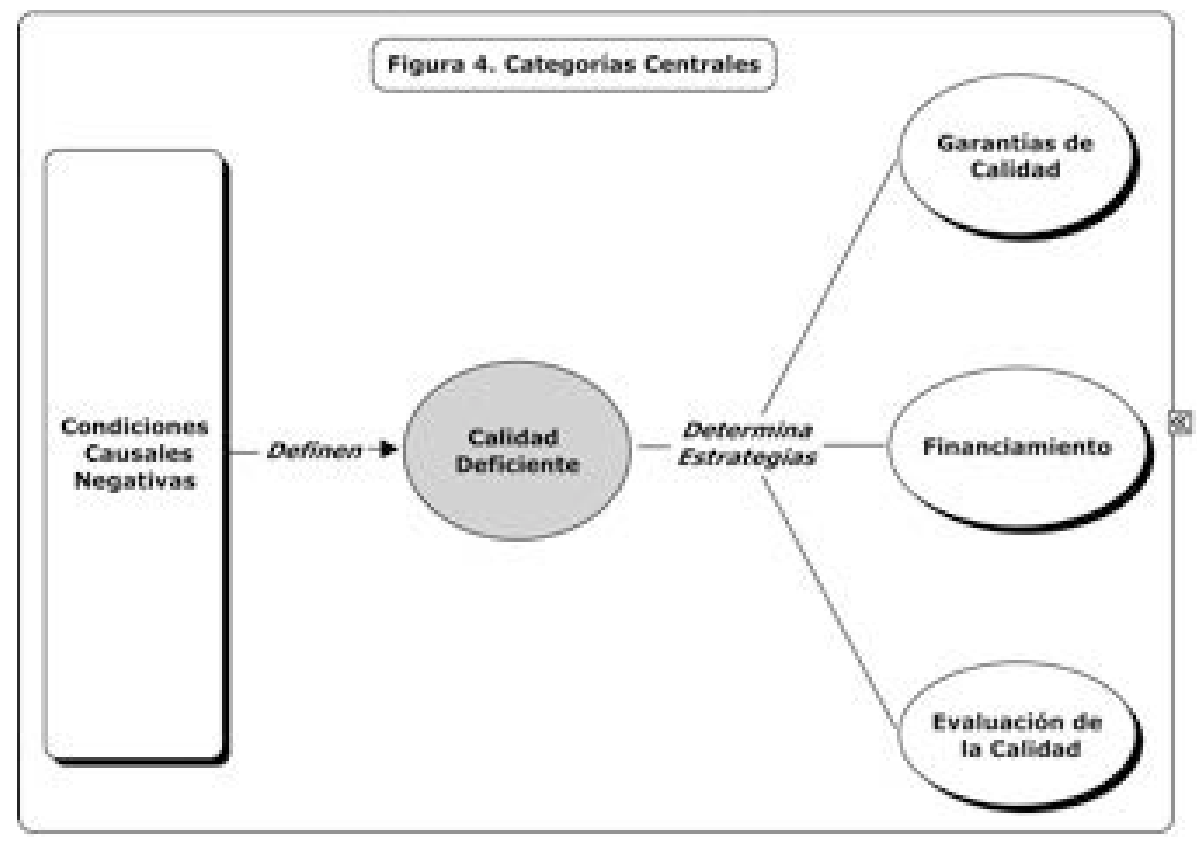

Luego de realizar el análisis de relaciones condicionadas, en conjunto con un análisis de frecuencia de unidades de sentido por familias de códigos, es posible identificar las categorías centrales del fenómeno (Fig.4). El modelo preliminar tiene como base una categoría general que, expresada en las condiciones causales del fenómeno, indica que la calidad de la educación en Chile es deficiente. A partir de estas condiciones causales, se propone estrategias que se organizan en torno a tres categorías principales: Garantías de calidad, Financiamiento y Evaluación.

\section{CONCLUSIONES Y DISCUSIÓN}

Se presentan en el presente artículo el resultado de la codificación axial realizado mediante la teoría-metodología fundamentada, respecto a las representaciones sociales en torno a calidad y sus factores de mejoramiento. El modelo preliminar de relaciones otorga algunas pistas respecto a las relaciones y niveles de importancia descriptiva y explicativa que las categorías y la interpretación hicieron emerger basado en un método riguroso de comparación constante entre dos investigadores.

Los resultados obtenidos permiten comprender los aspectos que construyen las RS sobre calidad educativa en los actores sociales en el marco del conflicto estudiantil ocurrido durante el año 2011. Independientemente de las diversas posturas y los énfasis particulares de diversos sectores y/o actores, es posible distinguir 3 niveles discursivos, 
que estructuran el debate sobre calidad de la educación y las RS que lo fundan. En un primer nivel, se encuentra un discurso evaluativo que en el modelo explicativo aparece como condiciones causales, que se orienta a juzgar la calidad de la educación y en el que se observa un alto nivel de congruencia en los actores, en el sentido de señalar que esta es insuficiente o abiertamente deficitaria. El siguiente nivel que está asociado a las estrategias y acciones que develan con medios para lograr la calidad, apareciendo tres elementos centrales: garantía, financiamiento y evaluación. El último nivel tiene que ver con principios y aspiraciones sociales mayores que dan el marco ideológico a la discusión sobre calidad. Se advierte que, en todos estos niveles, existen discursos de los actores analizados que se construyen en relación con un saber experto que tiene diversos orígenes y que, al ser expuestos en el debate público, presenta ciertas similitudes y diferencias.

El modelo explicativo que se ha construido tiene carácter preliminar, debido a que no ha sido posible llegar a la saturación teórica y el análisis sólo cumplió con dos de los 3 pasos propuestos por la Teoría Fundamentada: la codificación abierta y la axial. En este modelo se han identificado 3 categorías centrales que identifican los factores que son asociados a la calidad de la educación. El primero corresponde a las garantías de una educación de calidad que incorpora la discusión respecto al rol del Estado y cuáles son los mecanismos que este emplea para dar curso a esta garantía. Aparece la pregunta por quién garantiza la calidad de la educación en Chile, dado el sistema de provisión mixto y el sistema de financiamiento a la educación escolar basado en el subsidio a la demanda. El segundo incorpora diversos elementos vinculados al financiamiento, que considera la discusión respecto a las bases estructurales del sistema de financiamiento de la educación escolar y universitaria, por los montos necesarios para financiar una educación de calidad y las discusiones en torno a lucro y calidad. El tercero se refiere a la evaluación, que se expresa en diversos niveles, desde la evaluación de aprendizajes en el aula hasta los mecanismos de monitoreo y evaluación de la calidad a nivel del sistema.

Un tema relevante a discutir y que debiera ser materia de estudios posteriores con una mayor cantidad de fuentes, consiste en saber en qué medida aspectos que aparecen como condiciones y como cursos de acción en los discursos de los actores están determinando las RS acerca de calidad educativa. Si bien se entiende que la aspiración por mejorar la calidad está en el horizonte de las demandas estudiantiles y de la discusión sobre educación, en el proceso en el que se ha construido el debate no es claro si las discusiones sobre aspectos como lucro, gratuidad, sistema de financiamiento y acceso se vinculan directamente a la aspiración de calidad, propiamente tal o bien se instalan en una discusión por principios y valores que están sobre la calidad: igualdad, derechos, cambio social.

Se espera que, una vez alcanzada la saturación teórica del cuerpo de documentos y temáticas analizadas, se pueda explorar un nivel más profundo de relaciones que nos permitan sondear empíricamente si los niveles de correspondencia de las categorías de calidad y ciertos factores de calidad se circunscriben en mayor o menor medida a algunas de las Nociones y fines de la educación descritos. La comprensión de estas relaciones otorga un camino que posibilita la distinción de las demandas pro-movimiento estudiantil y las respuestas pro-gobierno en un contexto tensionado, además, por lineamientos a favor de transformaciones y otros de carácter estabilizador. Por otro lado, consideramos relevante aplicar este tipo de metodologías dado que, por medio del proceso inductivo de reconstrucción de códigos y categorías, la interpretación y comparación constante 
permite identificar los sentidos socialmente construidos, haciendo emerger la estructura interna de la RS (Umaña, 2002). Esto es especialmente importante para el establecimiento de principios de enfoque al analizar discursos públicos que integran el sentido común, pero, al mismo tiempo la influencia ideologizada o no del campo académico. Dadas las limitaciones temporales de la selección del corpus académico a un período de tres meses, sería interesante identificar cómo podrían variar estas expresiones antes y después del conflicto, ya que, como hipótesis de base, se esperaría que las posiciones de ciertos actores en distintos medios de prensa respecto a la temática se defina más, al haber mayor consolidación del debate y cierto grado de movimiento en algunas RS para algunos actores particulares.

Finalmente, cabe agregar que, asimismo una vez alcanzada la saturación teórica, sería deseable aventurar una nueva hipótesis respecto a las relaciones que podrían tener ciertas aspiraciones de transformación del sistema educativo con ciertas nociones de educación, calidad y factores de mejoramiento; lo cual podría expresar de manera diferenciada los discursos pro gobierno y pro movimiento estudiantil.

\section{REFERENCIAS BIBLIOGRÁFICAS}

Bourdieu, P. (1988). La Distinción. Criterio y bases sociales del gusto. México: Taurus.

Braslavsky, C. y Cosse, G. (1996). Las actuales Reformas Educativas en América Latina: Cuatro Actores, Tres Lógicas y Ocho Tensiones. Santiago de Chile: PREAL.

Cameron, K. \& Whetten, D. (1983). Organizational effectiveness: A comparison of multiple models. New York: Academic Press.

Flores, R. (2009). Observando a observadores: Una introducción a las técnicas cualitativas de investigación social. CIP. Santiago de Chile: Ediciones Universidad Católica de Chile.

Freire, P. (1969). La educación como práctica de la libertad (1ª ed.). Madrid: Siglo XXI.

Glaser, B. G., \& Strauss, A. (1967). The discovery of grounded theory: Strategies for qualitative research. Chicago: Aldine.

Goulding, C. (2005). Grounded theory, ethnography and phenomenology. A comparative analysis of three qualitative strategies for marketing research. European Journal of Marketing, 39, 3/4, 294-308.

Howart, C. (2006). A social representation is not a quiet thing: Exploring the critical potential of social representations theory. British Journal of Psychology, 45, 65-86.

Khan, M (2011). Electronic media and interpersonal discourse: Mediation in Crises. European Journal of Social Sciences, 18, 4, 553-559.

Martinic, S. (2006). El estudio de las representaciones sociales y el análisis estructural del discurso. En Canales, M. (Ed.), Metodologías de investigación social (pp. 299-319). Santiago: Lom.

Moscovici, S. (1986). La Representación Social: Fenómenos, concepto y teoría. En Psicología Social II. Barcelona. España: Paidós.

Oliver, C. (2011). Critical Realist Grounded Theory: A New Approach for Social Work Research. British Journal of Social Work 1-17.

Ottone, E. \& Hopenhayn M. (2007). Desafíos educativos ante la sociedad del conocimiento. Revista Pensamiento Educativo, 40, 1, 12-30.

Reeves, C. \& Bednar D. (1994). Defining Quality: Alternatives and Implications. Academy of Management Review, 19,3, 419-445.

Strauss, A \& Corbin, J. (2002). Bases de la investigación cualitativa. Técnicas y procedimientos para desarrollar la teoría fundamentada. Antioquia: Editorial Universidad de Antioquia. 
Suarez, L. (1999). El papel de los medios de comunicación en la construcción de las representaciones sociales en torno a la inseguridad ciudadana. Espacio Abierto, 8, 3, 389-401.

Umaña, S. (2002). Las representaciones sociales. Ejes Teóricos para su discusión. Cuaderno De Ciencias Sociales 127. San José, Costa Rica: FLACSO.

UNESCO (2005). Educación para todos: El imperativo de la Calidad. Informe de seguimiento de la Educación para Todos en el Mundo. Paris: UNESCO. Disponible en www.unesco.org/education.

UNESCO (2007). Educación de Calidad para Todos: un asunto de derechos humanos. Santiago de Chile: OREALC/UNESCO.

UNESCO (2008). Quality education, Equity and Sustainable Development: A holistic vision through UNESCO's four World Education Conferences 2008-2009.

UNICEF (2000). Defining Quality in Education. Working paper series. Education section. NY: United Nations Children's Fund.

Vasilachis de Gialdino, I. (Coord.). (2006). Estrategias de Investigación Cualitativa. Barcelona: Gedisa.

Wilson, K \& Howell, D. (2008). Clarifying Analysis and Interpretation in Grounded Theory: Using a Conditional Relationship Guide and Reflective Coding Matrix. International Journal of Qualitative Methods, 7, 2, 2-15. 
